\title{
A CHARACTERIZATION OF COVERING DIMENSION FOR COLLECTIONWISE NORMAL SPACES
}

\author{
JAMES AUSTIN FRENCH
}

Ostrand [3] gave the following characterization of the covering dimension for metric spaces. A metric space $X$ is of dimension $\leqq n$ if and only if for each open cover $C$ of $X$ and each integer $k \geqq n+1$ there exist $k$ discrete families of open sets $U_{1}, U_{2}, \cdots, U_{k}$ such that the union of any $n+1$ of the $U_{i}$ is a cover of $X$ which refines $C$. The purpose of this paper is to generalize Ostrand's theorem to collectionwise normal spaces by using an argument similar to that of Michael [1, Theorem 2].

Definitions. The collection $H$ of subsets of the topological space $X$ strongly refines the collection $G$ of subsets of $X$ if and only if for each $h \in H$ there is an element $g \in G$ such that $\bar{h} \subset g$, where $\bar{h}$ denotes the closure of $h$.

The collection $H$ of subsets of the topological space $X$ is $\sigma_{n}$-discrete if and only if $H$ is the union of $n$ discrete collections.

Suppose $X$ is a topological space. The covering dimension of $X$ is -1 , denoted $\operatorname{dim} X=-1$, if and only if $X$ is empty. If $n$ is a nonnegative integer, then the covering dimension of $X$ is less than or equal to $n$, denoted $\operatorname{dim} X \leqq n$ if and only if for each open cover $G$ of $X$ there is an open cover $H$ of $X$ such that $H$ refines $G$ and ord $H \leqq n+1$.

REMARK. The usual definition for covering dimension is in terms of finite open covers. The above definition is equivalent to the usual definition of covering dimension for paracompact spaces. (See Theorem II.6, p. 22, of [2] and note that this theorem is true for any normal space and hence for any paracompact space.)

If $X$ is a topological space, $M \subset X$, and $G$ is a collection of subsets of $X$, then ord ${ }_{M} G=\operatorname{lub}\left\{\right.$ ord $\left.{ }_{p} G: p \in M\right\}$ where ord ${ }_{p} G$ is the number of elements of $G$ containing $p$. Also, ord ${ }_{x} G$ will be denoted ord $G$.

Notations. If $H$ is a collection of sets, then $\pi H$ denotes the common part of the elements of $H$ and $H^{*}$ denotes the union of the elements of $H$.

Theorem 1. If $X$ is a collectionwise normal space, $M$ is a closed subset of $X, n$ is a positive integer, $G$ is a collection each element of which is an open subset of $X$, and every point of $M$ is in exactly $n$ elements of $G$, then there is a discrete collection $H$ of open subsets of $X$ such that $H$ covers $M$ and strongly refines $G$.

Received by the editors June 24, 1969. 
Proof of Theorem 1. Assume hypothesis. For each $p \in M$ let $R(p)=\{g \in G: p \in g\}$. If $p \in M$, then $R(p)$ has exactly $n$ elements. Let $C=\{M \cap[\pi R(p)]: p \in M\}$.

It will now be shown that for all points $p, q \in M$ either $M \cap[\pi R(p)]$ $=M \cap[\pi R(q)]$ or $(M \cap / \pi R(p)]) \cap(M \cap[\pi R(q)])=\varnothing$ from which it follows that $C$ is a discrete collection of closed point sets. Assume each of $p, q, t \in M$ and $t \in\{(M \cap[\pi R(p)]) \cap(M \cap[\pi R(q)])\}$. Each of $R(p)$ and $R(q)$ has exactly $n$ elements. Each element of $R(p) \cup R(q)$ contains $t$, and $t$ is contained by only $n$ elements of $G$. Hence, $R(p)$ $=R(q)$. Obviously $M \cap[\pi R(q)]=M \cap[\pi R(p)]$.

There is a discrete collection $H$ of open sets such that each element of $C$ is a subset of an element of $H$, no element of $H$ intersects two elements of $C$, and $H$ strongly refines $G$. $H$ covers $M$ since $M$ is $C^{*}$.

THEOREM 2. If $X$ is a collectionwise normal space, $M \subset X, M$ is closed, $n$ is a nonnegative integer, $G$ is a collection of open subsets of $X$ covering $M$, and ord ${ }_{M} G \leqq n+1$, then there is a collection $H$ of open subsets of $X$ such that $H$ covers $M, H$ strongly refines $G$, and $H$ is $\sigma_{n+1^{-}}$ discrete.

Proof of Theorem 2. By induction. The proposition is true for $n=0$ by Theorem 1 . Assume that the proposition is true for the nonnegative integer $n$. Assume the proposition hypothesis for the nonnegative integer $n+1$. Let $R(p)=\{g \in G: p \in g\}$ for each $p \in M$. Let $A=\{p \in M: n+2$ elements of $G$ contain $p\}$. If $p \in A$, then $M \cap[\pi R(p)]$ is open with respect to $M$ and $M \cap[\pi R(p)] \subset A$. So $A=\{M \cap[\pi R(p)]: p \in A\}\}^{*}$ and hence $A$ is open with respect to $M$. Now $M-A$ is closed and ord ${ }_{M-A} G \leqq n+1$. By the induction hypothesis there is a collection $K$ of open subsets of $X$ that covers $M-A$, strongly refines $G$, and is $\sigma_{n+1}$-discrete. Now $M-K^{*}$ is closed and $M-K^{*} \subset A$. So by Theorem 1 there is a discrete collection $R$ of open subsets of $X$ that covers $M-K^{*}$ and strongly refines $G . K \cup R$ is a collection of open subsets of $X$ that covers $M$, strongly refines $G$, and is $\sigma_{n+2}$-discrete.

Theorem 3. The collectionwise normal space $X$ has $\operatorname{dim} \leqq n$ if and only if for each collection $G$ of open sets covering $X$ there is a collection $H$ of open sets that covers $X$, strongly refines $G$, and is $\sigma_{n+1}$-discrete.

Proof of Theorem 3. The "if" part of the proof is obvious. The "only if" part of the proof will now be given. Suppose $X$ is a collectionwise normal space and $\operatorname{dim} X \leqq n$. Assume $G$ is a collection of open sets covering $X$. There is a collection $K$ of open sets such that $K$ covers $X, K$ refines $G$, and ord $K \leqq n+1$. Now $X$ is a closed subset of 
$X$ so by Theorem 2 there is a collection $H$ of open sets such that $H$ covers $X, H$ strongly refines $K$, and $H$ is $\sigma_{n+1}$-discrete.

THEOREM 4. The collectionwise normal space $S$ has $\operatorname{dim} \leqq n$ if and only if for each open cover $C$ of $S$ and each integer $k \geqq n+1$ there exist $k$ discrete families of open sets $U(1), U(2), \cdots, U(k)$ such that the union of any $n+1$ of the $U(i)$ is a cover of $S$ which strongly refines $C$.

Proof of Theorem 4. The proof of the "if" part of the theorem is obvious. The proof of the "only if" part will now follow by induction. By Theorem 3, the proposition is true for $k=n+1$. Assume that the proposition is true for the integer $k \geqq n+1$. There exist $k$ discrete families of open sets $U(1), U(2), \cdots, U(k)$ such that the union of any $n+1$ of the $U(i)$ is a cover of $S$ which strongly refines $C$. Let $A=\{x: x$ is an $n$-termed subsequence of $1,2, \cdots, k\}$. For each $x \in A$ let $H_{x}=S-[U(x(1)) \cup U(x(2)) \cup \ldots \cup U(x(n))]^{*}$; hence, $H_{x}$ is closed. Let $E=\left\{H_{x}: x \in A\right\}$. It will now be shown that if $H_{x}$ and $H_{y}$ are two nonempty elements of $E$, then $H_{x}$ does not intersect $H_{y}$. Suppose $H_{x}$ and $H_{y}$ are two nonempty elements of $E$ that intersect. Thus $x$ is not $y$. There is a $p \in S-[U(x(1)) \cup \cdots \cup U(x(n))]^{*}$ and $p \in S-[U(y(1)) \cup \ldots \cup U(y(n))]^{*}$. There is a positive integer $j$ such that $1 \leqq j \leqq n$ and $y(j)$ is not a term of $x$. Thus,

$$
p \notin[U(y(j)) \cup U(x(1)) \cup U(x(2)) \cup \cdots \cup U(x(n))]^{*}
$$

but it is an open set collection covering $S$. Contradiction.

Now $E$ is a finite collection of mutually exclusive closed point sets, so $E$ is discrete. For each $x \in A$, let $x^{\prime}$ be a positive integer such that $1 \leqq x^{\prime} \leqq k$ and $x^{\prime}$ is not a term of $x$. $U\left(x^{\prime}\right)$ must cover $H_{x}$. For each $x \in A$ let $E_{x}=\left\{h \cap H_{x}: h \in U\left(x^{\prime}\right)\right\}$ and note that $E_{x}$ is a discrete refinement of $U\left(x^{\prime}\right)$ each element of which is closed. Let $T=\left\{E_{x}: x \in A\right\}^{*}$. Now $T$ is a discrete refinement of $U(1) \cup U(2) \cup \ldots \cup U(k)$ each element of which is closed. Hence $T$ refines $C$. There is a discrete collection $U(k+1)$ of open sets that strongly refines $C$ and covers $T^{*}$. Note that $T^{*}=E^{*}$. Now consider the sequence $U(1), \cdots, U(k+1)$ and suppose that $y$ is a subsequence of $1,2, \cdots, k+1$ with only $n+1$ terms. If $k+1$ is not a term of $y$, then by the induction hypothesis, $U(y(1)) \cup \ldots \cup U(y(n+1))$ is a cover of $S$ that strongly refines $C$. Suppose $k+1$ is a term of $y$. Let $x$ be the $n$-termed sequence such that $x(i)=y(i)$ for $i=1,2, \cdots, n$. Now $H_{x} \subset E^{*}$. Thus $U(k+1)$ covers $H_{x}$. So $U(y(1)) \cup \ldots \cup U(y(n+1))$ covers $S$ and strongly refines $C$.

Corollary 1. The metric space $X$ has Ind $\leqq n$ if and only if for each open cover $G$ of $X$ and each integer $k \geqq n+1$ there exist $k$ discrete families 
of open sets $U(1), U(2), \cdots, U(k)$ such that the union of any $n+1$ of the $U(i)$ is a cover of $X$ which strongly refines $G$ and if $b$ is the boundary of an element of $U(i)$, then Ind $b \leqq n-1$.

Ind denotes large inductive dimension. The proof of Corollary 1 follows easily from Theorem 4 and the fact the dim is equivalent to Ind in metric spaces.

\section{REFERENCES}

1. E. Michael, Point-finite and locally finite coverings, Canad. J. Math. 7 (1955), 275-279. MR 16, 1138.

2. J. Nagata, Modern dimension theory, Bibliotheca Math., vol. 6, Interscience, New York, 1965. MR 34 \#8380.

3. P. A. Ostrand, Dimension of metric spaces and Hilbert's problem 13, Bull. Amer. Math. Soc. 71 (1965), 619-622. MR 31 \#1654.

Auburn University, Auburn, Alabama 36830 and

Louisiana State University, Baton Rouge, Louisiana 70803 